Journal of Sustainable Agricultural Sciences
http://jsas.journals.ekb.eg/

\title{
Evaluation of Six Insecticides for the Control of Potato Whitefly (Bemisia tabaci) in Relation to Induced Resistance and Tuber Quality
}

Farid S. Sabra ${ }^{1}$, Mona A. A. Mahmoud ${ }^{2}$, Rania S. Ammar ${ }^{2}$ and Soad M. Ahmed ${ }^{\text {* }}$ Pesticide Chemistry and technology Department, Faculty of Agriculture, Alexandria University, Elshatby,Alexandria, Egypt

${ }^{2}$ Plant Protection research Institute, Agriculture Research Center (ARC), Alexandria, Egypt

$\mathbf{P}$ OTATO whitefly, Bemisia tabaci (Homoptera: Aleyrodidae) is one of the most important pests of potato. Field experiments were carried out during two successive growing winter seasons 2017/2018 and 2018/2019 at the Research Station of Faculty of Agriculture Alexandria University, to evaluate the efficacy of six conventional insecticides, emamectin benzoate + thiamethoxam, imidacloprid, etofenprox, fenitrothion, pymetrozine and thiamethoxam against Bemisia tabaci on potato plants (Solanum tuberosum L.) of variety sponta, either as direct effect or indirect effect during induced resistance and their relationship with crop yield. Results showed that emamectin benzoate+thiamethoxam, thiamethoxam and imidacloprid achieved high reduction percentage of whitefly adults. Also, fenitrothion, pymterozine and imidacloprid achieved high reduction percentage of whitefly immature. Fortunately, these treatments had a positive effect, both on the measures of induced resistance that were estimated, and at the same time they had a promising effect on the potato yield in terms and quality.

Keywords: Bemisia tabaci, Potato plants, Insecticides, Induced resistance, Yield, Quality

\section{Introduction}

Potato crop is considered one of the most important vegetable crops, and plays an important role in the Egyptian diet, also as an export vegetable crop. Potato plants Solanum tuberosum are attacked by many insect species which cause serious injury and thus the final yield is reduced. Whitefly, Bemisia tabaci (Gennadius) is a complex cryptic species and destructive insect pest which is reported to attack and damage about 600 plant species (Nombelaand Muniz, 2010). The damages inflicted by whitefly on its plant hosts result directly from sap sucking and toxin injection, and indirectly from virus transmission and honeydew secretion, which serves as a substrate for the growth of sooty mold fungi upon the leaves (Hirose et al. 2015 and Arnemann et al. 2019). Whitefly is among the most serious pests in cultivation of potatoes, since it has a significant influence on yield (Palumbo 2019 and Bhatnagar, 2007).
Insecticides especially neonicotinoid insecticides are applied to plants in managed landscapes worldwide and it is very likely that the insecticidemediated disruption of plant defenses that documented is widespread (Szczepaniec et al. 2013; Ford et al. 2010; Karthikeyan et al. 2009). Many plant-protecting compounds have been identified that combine both directaction on the pathogen and priming-inducing activity in the plant, and several have given rise to new commercial formulations such as the insecticide Imidacloprid (Eyles et al., 2010). The enhanced biosynthesis and accumulation of proteins are integral components of the induced chemical defense system against insects (Keeling and Bohlmann 2006; Franceschi et al. 2005).

Phenolic compounds are a major class of inducible defense compoundsin many plants (Witzell and Martin and 2008; Tsai et al. 2006). Recent evidence suggests that correlations between insect performance and concentrations of phenolic 
compounds (Ruuhola et al. 2008). Oxidation of phenolic compounds produces reactive oxygen species, resulting in oxidative stress inmidgut tissues (Barbehenn et al. 2009).It is now generally accepted that plant phenolics are secondary metabolites involved in the defense mechanism of plants against insect (Lattanzio et al., 2006). Pesticide residues may interfere with biochemical and physiological processes in plants retarding the growth and yield of the plant. They may also lower its food quality and prevent its use as food by affecting its quality characteristics (Chauhan et al., 2013).

Induced resistance can be exploited for developing crop cultivars, which readily produce the inducible response upon mild infestation and can act as one of components of integrated pest management for sustainable crop production.Ultimately, to establish an effective management strategy for potato whitefly pest.

\section{Experimental Materials and Methods}

Field trials and experimental design for the insecticidal activity

Field experiments were conducted at the Research Station of Faculty of Agriculture, Alexandria University, during two successive growing winter seasons 2017/2018 and 2018/2019 on potato plants (Solanum tuberosum L.) of variety Sponta. Treatments were arranged in a randomized complete block design (RCBD). Each treatment was replicated three times (42 $\mathrm{m}^{2}$ each). The normal agricultural practices were applied. The insecticides were sprayed by Knapsack sprayer equipment (CP3) at the rate of 200 liter per feddan. Control was sprayed only by water. Six insecticides were used in this experiment, trade names, common names, companies and rates were introduced in Table 1.

The inspection samples of whitefly adult individuals were carried out before treatment and 1day (initial effect), 3, 5, 7, 10 days post treatment (residual effect). Counts were done in the early morning when flight activity is minimal according to Bulter et al. (1988). Five leaves of each plot were picked from lower, middle and upper of plant and put in numbered paper bags and transferred to the laboratory for examining the immature stages on the upper and lower surfaces by using stereoscopic binocular microscope. The reduction percentages in numbers of whitefly adults and immature stages were calculated in accordance to Henderson and Tilton's equation (1955). The treatments were compared with each other using onway ANOVA with $\mathrm{LSD}_{0.05}$ (CoStat Statistical Software, 1990).

The effect on some induced resistance parameters of potato leaves

Dry matter content

The dry matter content of potato tubers was calculated from the following formula:

$$
\text { D.M.C }=[100-(\text { F.Wt.-D.Wt.)/20]*100) }
$$

where: F.Wt. = tuber sample weight before drying, D.Wt. = tuber sample weight after drying on $105^{\circ} \mathrm{Cuntilthe}$ Constant weight.

Total soluble phenol content assay

Total soluble phenol content of potato leaves, was extracted according to Ahmed (2010) and determined according to Slinkard and Singleton (1997). Total soluble phenol content was standardized against tannic acid and absorbance values were converted to $\mu \mathrm{g}$ of phenols per gram of fresh weight potato leaves.

\section{Total protein assay}

Total protein was determined according to method described by Bradford (1976), with slight modification proposed by Dixon (1985). The developed color was measured at $595 \mathrm{~nm}$. The reading was related to standard curve of BSA (Bovine Serum Albumin) protein.

TABLE 1. Tested insecticides againstbemisiatabaci on potato plants

\begin{tabular}{llll}
\hline \multicolumn{1}{c}{ Common name } & \multicolumn{1}{c}{ Trade name } & \multicolumn{1}{c}{ Company } & \multicolumn{1}{c}{ Rate/feddan } \\
\hline $\begin{array}{l}\text { Etofenprox } \\
\text { Pymetrozine }\end{array}$ & Primo $^{\circledR} 10 \% \mathrm{SC}$ & Shanxi Lvhai Agrochemicals, China & $188 \mathrm{~cm}^{3}$ \\
$\begin{array}{l}\text { Emamectin benzoate }+ \\
\text { thiamethoxam }\end{array}$ & Tedo $^{\circledR} 50 \% \mathrm{WDG}$ & Syngenta & $50 \mathrm{~g} / 100 \mathrm{liter}$ \\
$\begin{array}{l}\text { Thiamethoxam } \\
\text { Fenitrothion }\end{array}$ & Agriflex $^{\circledR} 18.56 \% \mathrm{Sc}$ & Syngenta & $250 \mathrm{~cm}^{3}$ \\
midacloprid & Vitara $^{\circledR} 25 \% \mathrm{WDG}$ & BR agrotech limited -India & $30 \mathrm{~g} / 100$ liter \\
\hline
\end{tabular}




\section{The effect on potato tuber quality characters}

Total tuber yield

In order to determine the side effects of the treatments total yield of potato tuber. All tubers of each plot were harvested. Weighted and recorded as ton /Fadden. Then the percentage of increasing of the total yield was calculated.

\section{Total soluble solid (TSS)}

A total soluble solid (TSS) in the fresh potato tubers sap was done using a digital refractometer (Abd El-Latif et al., 2011).

Ascorbic acid in fresh potato tubers

Potato tuber were extracted with $0.4 \%$ oxalic acid solution. Spectrophotometric determination of ascorbic acid was carried out according to Sabra 1993, Egoaville et al., 1988 and Burgos et al., 2009.

\section{Total acid of potato tubers}

Samples of potato tubers were blended with distilled water $(1: 2.5)$. The total acidity as citric acid was determined according Sabra, 1993.

\section{Polyphenols in fresh potato tubers}

Extraction of polyphenols was carried out according to Zarzecka and Gugata (2011). The method of determination based on the estimation of the color resulted from the reaction between the FolinCicoalteu reagent and hydroxyl group of phenolic compounds under alkaline condition, according to Sabra (1993), Waterhouse (2002) and Burgos et al. (2013).

\section{Statistical analysis}

The data of in vivo experiments were analyzed by Two-way analysis of variance (ANOVA). Mean separations were performed by LSD test and differences at $\mathrm{P}<0.05$ were considered as significant (SAS Software).

\section{Results and Discussion}

Efficacy of tested insecticides on whitefly adults According to insecticides application, emamectin benzoate + thiamethoxam, imidacloprid, etofenprox, fenitrothion, pymetrozine and thiamethoxam in season of 2017/2018, population of whitefly adults were significantly reduced, (Table 2). Data in the first spray showed that there were no significant differences between the six insecticides after 1day post treatment (initial reduction). Concerning to the residual reduction, imidacloprid and thiamethoxam were the most effect recording $79.2 \%$ and $73.9 \%$. Etofenprox was the least effect (56.6\%).In the second spray, data indicated that emamectin benzoate+thiamethoxam gave the highest initial reduction percentage $(96.0 \%)$ followed by imidacloprid $83.2 \%$. No significant differences between all insecticides on initial reduction of $b$. tabaci adults. The general mean of the first and second spray indicated emamectin benzoate+thiamethoxam achieved high initial and residual reduction percentage $(82.3 \%$, $68.5 \%$ ) followed by imidacloprid $(75.2 \%, 68.0 \%)$. Concerningtoseason $2018 / 2019$, in the first spray, data in (Table 3) indicated that thiamethoxam and emamectin benzoate+thiamethoxam achieved the highest initial and residual reduction percentage $(76.9 \%, 71.0 \%)$ and $(76.0 \%, 68.3 \%)$. During the second spray emamectin benzoate+thiamethoxam was the most effect achieving $90.4 \%$ initial reduction followed by imidacloprid $89.8 \%$. Regarding the residual effect, imidacloprid recorded the highest residual reduction percentage $82.5 \%$, there were no significant differences between the other insecticides. The general mean of the first and second spray showed that emamectinbenzoate+thiamethoxam gave the highest initial reduction percentage $83.2 \%$ followed by thiamethoxam $79.2 \%$. Also, imidacloprid gave high residual reduction percentage $73.5 \%$. Pymetrozine and etofenprox recorded low residual reduction percentage $59.9 \%$ and $58.4 \%$.

TABLE 2. Mean reduction percentagesof B.tabaci adults on potato plants during season of 2017/2018

\begin{tabular}{|c|c|c|c|c|c|c|c|c|c|c|c|c|}
\hline \multirow{4}{*}{ Insecticides } & \multicolumn{12}{|c|}{$\%$ Reduction after different times of treatment } \\
\hline & \multicolumn{5}{|c|}{$1^{\text {st }}$ spray } & \multicolumn{5}{|c|}{$2^{\text {nd }}$ spray } & \multicolumn{2}{|c|}{$\begin{array}{l}\text { General mean of } 1^{\text {st }} \\
\text { and } 2^{\text {nd }} \text { spray }\end{array}$} \\
\hline & \multirow{2}{*}{$\begin{array}{l}1 \text { day } \\
\text { (initial) }\end{array}$} & \multicolumn{3}{|c|}{ Residual } & \multirow{2}{*}{$\begin{array}{l}\text { Mean of } \\
\text { residual }\end{array}$} & \multirow{2}{*}{$\begin{array}{c}1 \text { day } \\
\text { (initial) }\end{array}$} & \multicolumn{3}{|c|}{ Residual } & \multirow{2}{*}{$\begin{array}{l}\text { Mean of } \\
\text { residual }\end{array}$} & \multirow{2}{*}{$\begin{array}{c}1 \text { day } \\
\text { (initial) }\end{array}$} & \multirow{2}{*}{ Residual } \\
\hline & & 3day & 7day & 10day & & & 3day & 7 day & 10day & & & \\
\hline Etofenprox & $43.3^{\mathrm{a}}$ & 50.6 & 71.6 & 47.7 & $56.6^{\mathrm{b}}$ & $72.0^{\mathrm{c}}$ & 54.4 & 59.1 & 58.7 & $57.4^{\mathrm{a}}$ & $57.7^{\mathrm{b}}$ & $57.0^{\mathrm{b}}$ \\
\hline Pymetrozine & $53.9^{\mathrm{a}}$ & 77.1 & 75.9 & 26.4 & $59.8^{\mathrm{ab}}$ & $78.1^{\mathrm{bc}}$ & 74.8 & 55.5 & 61.1 & $63.8^{\mathrm{a}}$ & $66.0^{\mathrm{b}}$ & $61.8^{\mathrm{ab}}$ \\
\hline Emamectinbenzoate + Thiamethoxam & $68.6^{\mathrm{a}}$ & 91.5 & 75.9 & 42.5 & $69.9^{\mathrm{ab}}$ & $96.0^{\mathrm{a}}$ & 97.6 & 51.5 & 52.2 & $67.1^{\mathrm{a}}$ & $82.3^{\mathrm{a}}$ & $68.5^{\mathrm{a}}$ \\
\hline Thiamethoxam & $69.9^{\mathrm{a}}$ & 76.7 & 82.7 & 62.4 & $73.9^{\mathrm{a}}$ & $77.5^{\mathrm{bc}}$ & 62.3 & 53.7 & 52.1 & $56.0^{\mathrm{a}}$ & $73.7^{\mathrm{ab}}$ & $65.0^{\mathrm{ab}}$ \\
\hline Fenitrothion & $63.4^{\mathrm{a}}$ & 80.5 & 76.6 & 43.6 & $66.9^{\mathrm{ab}}$ & $79.7^{\mathrm{bc}}$ & 55.6 & 50.0 & 50.9 & $52.2^{\mathrm{a}}$ & $71.5^{\mathrm{ab}}$ & $59 . .^{\text {ab }}$ \\
\hline Imidacloprid & $67.2^{\mathrm{a}}$ & 70.3 & 86.1 & 81.1 & $79.2^{\mathrm{a}}$ & $83.2^{\mathrm{b}}$ & 62.5 & 48.5 & 59.7 & $56.9^{\mathrm{a}}$ & $75.2^{\mathrm{ab}}$ & $68.0^{\mathrm{ab}}$ \\
\hline
\end{tabular}

Different letters indicate significant differences among treatments within the same column according to least significant difference test $(\mathrm{P}=0.05)$. 
TABLE 3. Mean reduction percentages of B.tabaci adults on potato plants during season of 2018/2019

\begin{tabular}{|c|c|c|c|c|c|c|c|c|c|c|c|c|}
\hline \multirow[t]{2}{*}{ Insecticides } & \multicolumn{5}{|c|}{$1^{\text {st }}$ spray } & \multicolumn{5}{|c|}{$2^{\text {nd }}$ spray } & \multicolumn{2}{|c|}{$\begin{array}{c}\text { General mean of } 1 \\
\text { and } 2^{\text {nd }} \text { spray }\end{array}$} \\
\hline & $\begin{array}{l}1 \text { day } \\
\text { (initial) }\end{array}$ & 3day & 7 day & 10 day & $\begin{array}{l}\text { Mean of } \\
\text { residual }\end{array}$ & $\begin{array}{l}1 \text { day } \\
\text { (initial) }\end{array}$ & 3 day & 7 day & 10 day & $\begin{array}{l}\text { Mean of } \\
\text { residual }\end{array}$ & $\begin{array}{l}1 \text { day } \\
\text { (initial) }\end{array}$ & Residual \\
\hline Etofenprox & $65.2^{\mathrm{c}}$ & 49.7 & 61.2 & 49.4 & $53.4^{\mathrm{c}}$ & $84.5^{\mathrm{bc}}$ & 65.1 & 58.0 & 66.9 & $63.3^{\mathrm{b}}$ & $74.8^{\mathrm{b}}$ & $58.4^{\mathrm{d}}$ \\
\hline $\begin{array}{l}\text { Emamectin } \\
\text { benzoate+Thiamethoxam }\end{array}$ & $76.0^{\mathrm{ab}}$ & 75.2 & 67.4 & 62.2 & $68.3^{\mathrm{a}}$ & $90.4^{\mathrm{a}}$ & 76.5 & 75.0 & 72.6 & $74.7^{\mathrm{ab}}$ & $83.2^{\mathrm{a}}$ & $71.5^{\mathrm{ab}}$ \\
\hline Thiamethoxam & $76.9^{\mathrm{a}}$ & 69.1 & 71.6 & 72.3 & $71.0^{\mathrm{a}}$ & $81.4^{c}$ & 71.3 & 65.3 & 65.2 & $67.3^{\mathrm{b}}$ & $79.2^{\mathrm{ab}}$ & $69.1^{\mathrm{ab}}$ \\
\hline Fenitrothion & $70.4^{\mathrm{bc}}$ & 79.9 & 57.6 & 40.1 & $59.2^{\mathrm{bc}}$ & $87.1^{\mathrm{abc}}$ & 71.5 & 69.3 & 72.4 & $71.0^{\mathrm{ab}}$ & $78.8^{\mathrm{ab}}$ & $65.1^{\mathrm{bc}}$ \\
\hline
\end{tabular}

Different letters indicate significant differences among treatments within the same column according to least significant difference test $(\mathrm{P}=0.05)$.

\section{Efficacy of tested insecticides on whitefly immature stages}

Data presented in (Table 4) showed that all insecticides reduced the number of immature stages of Bemisiatabaciin season of 2017/2018. In the first spray fenitrothion gave the highest initial and residual reduction percentage (94.8\% and $93.4 \%$ ), imidacoprid gave the least residual reduction percentage $70.4 \%$.In the second spray, initial reduction percentage indicated that the efficacy of the used insecticides on immature stages was not different significantly. The residual effect indicated that pymetrozine was the most effect giving $86.8 \%$, etofenprox was the least effect giving $64.8 \%$. The general mean of the first and the second spray indicated that fenitrothion gave the highest initial reduction percentage $87.3 \%$, no significant differences between pymetrozine, emamectin benzoate+thiamethoxam and imidacloprid which gave moderate reduction $75.4 \%, 74.4 \%$ and $73.0 \%$, respectively. The residual effect showed pymterozine was the best recording $89.4 \%$ followed by thiamethoxam $85.2 \%$, fentrothion $85.2 \%$, while etofenprox and imidacloprid was the least effect recording $75.8 \%$ and $73.8 \%$.In season of $2018 / 2019$, in the first spray, data in (Table 5) showed that fenitrothion gave the highest initial and residual reduction percentage $(91.6 \%$ and $90.0 \%)$. Also, emamectin benzoate+thiamethoxam gave the least initial reduction percentage $60.1 \%$, etofenprox gave the least residual reduction percentage $72.9 \%$.In the second spray, pymetrozine gave the highest initial percentage $87.0 \%$, fenitrothion was the least effect giving $77.2 \%$. Regarding the residual effect, imidacloprid was the best giving $83.5 \%$, while etofenprox was the least effect $75.0 \%$. The general mean of the first and second spray, after 1day post treatment, data indicated that fenitrothion recorded the highest initial reduction percentage $84.4 \%$ and emamectin benzoate+thiamethoxam was the least effect recording $71.9 \%$, concerning to the residual effect, imidacloprid and fenitrothion gave the highest reduction percentage $85.0 \%$ and $83.2 \%$, respectively, while etofenprox gave $73.9 \%$.

Results showed some insecticides were its effect high on adults of $B$. tabaci such as emamectinbenzoate + thiamethoxam, thiamethoxam and imidacloprid. Some achieved high reduction percentage against immature stages such as fenitrothion, pymterozine and imidacloprid which have different mode of action can use in sequence for controlling $b$. tabaci insects to avoid resistance of insecticides. The obtained results are in agreement with those of several investigators. $\mathrm{Nag}$ et al. (2018) found that the treatment Imidacloprid followed by Thiamethoxam at 15 days interval was most effective against whiteflies (1.56 per plant). El-Naggar and Zidan (2013) reported that treatments with imidacloprid and thiamethoxam as foliar applications were highly effective against aphids, up to 14 days in the case of jassids, while the effect was moderate on the whitefly population (mature and immature stages). Imidacloprid had more initial and residual effect than thiamethoxam against jassids. Kim et al. (2000) found that fenitrothion, imidacloprid and pymetrozine among insecticides achieved $>$ 95\% larvicidal activity on 3rd nymphal instars and $>95 \%$ adulticidal activity. The results are also supported by Preetha et al. (2009) who evaluated Imidacloprid and Thiamethoxam equally effective against whiteflies. Jambulkar et al., (2013) reported Imidacloprid most effective against whitefly. Ghosal and Chatterjee (2013) observed Imidacloprid superior against whitefly with lowest pest population and highest marketable yield. Similar results were reported by Arnemann et al. (2019), Afzal et al. (2014), Chandel et al. (2010) and Ananda et al. (2010). 
TABLE 4. Mean reduction percentages of $B$. tabaci immature stageson potato plants during season of $2017 / 2018$ $\%$ Reduction after different times of treatment

\begin{tabular}{|c|c|c|c|c|c|c|c|c|c|c|c|c|}
\hline \multirow[t]{3}{*}{ Insecticides } & \multicolumn{5}{|c|}{$1^{\text {st }}$ spray } & \multicolumn{5}{|c|}{$2^{\text {nd }}$ spray } & \multicolumn{2}{|c|}{$\begin{array}{c}\text { General mean of } 1^{\text {st }} \\
\text { and } 2^{\text {nd }} \text { spray }\end{array}$} \\
\hline & \multirow{2}{*}{$\begin{array}{c}1 \text { day } \\
\text { (initial) }\end{array}$} & \multicolumn{3}{|c|}{ Residual } & \multirow{2}{*}{$\begin{array}{l}\text { Mean of } \\
\text { residual }\end{array}$} & \multirow{2}{*}{$\begin{array}{c}1 \text { day } \\
\text { (initial) }\end{array}$} & \multicolumn{3}{|c|}{ Residual } & \multirow{2}{*}{$\begin{array}{l}\text { Mean of } \\
\text { residual }\end{array}$} & \multirow{2}{*}{$\begin{array}{c}1 \text { day } \\
\text { (initial) }\end{array}$} & \multirow{2}{*}{ Residual } \\
\hline & & 3 day & 7 day & 10 day & & & 3 day & 7 day & 10 day & & & \\
\hline Etofenprox & $82.2^{\mathrm{b}}$ & 92.8 & 89.3 & 78.7 & $86.9^{\mathrm{b}}$ & $84.7^{\mathrm{a}}$ & 63.1 & 79.7 & 51.7 & $64.8^{c}$ & $83.5^{\mathrm{ab}}$ & $75.8^{c}$ \\
\hline Pymetrozine & $71.1^{\mathrm{b}}$ & 91.2 & 90.2 & 95.0 & $92.1^{\mathrm{ab}}$ & $79.7^{\mathrm{a}}$ & 92.1 & 93.2 & 75.0 & $86.8^{\mathrm{a}}$ & $75.4^{\mathrm{b}}$ & $89.4^{\mathrm{a}}$ \\
\hline $\begin{array}{c}\text { Emamectin } \\
\text { benzoate+Thiamethoxam }\end{array}$ & $71.0^{\mathrm{b}}$ & 95.9 & 86.6 & 81.1 & $87.9^{\mathrm{b}}$ & $74.4^{\mathrm{a}}$ & 90.2 & 74.2 & 68.2 & $77.5^{\mathrm{b}}$ & $74.7^{\mathrm{b}}$ & $82.7^{\mathrm{b}}$ \\
\hline Thiamethoxam & $81.6^{\mathrm{b}}$ & 94.3 & 79.4 & 95.4 & $89.7^{\mathrm{ab}}$ & $76.8^{\mathrm{a}}$ & 82.9 & 85.7 & 73.3 & $80.6^{\mathrm{b}}$ & $79.2^{\mathrm{ab}}$ & $85.2^{\mathrm{ab}}$ \\
\hline Fenitrothion & $94.8^{\mathrm{a}}$ & 96.4 & 93.1 & 90.8 & $93.4^{\mathrm{a}}$ & $79.9^{\mathrm{b}}$ & 74.5 & 82.8 & 73.4 & $76.9^{\mathrm{b}}$ & $87.4^{\mathrm{a}}$ & $85.2^{\mathrm{ab}}$ \\
\hline Imidacloprid & $71.9^{\mathrm{b}}$ & 77.4 & 60.6 & 73.3 & $70.4^{\mathrm{c}}$ & $74.0^{\mathrm{a}}$ & 95.4 & 64.2 & 72.0 & $77.2^{\mathrm{b}}$ & $73.0^{\mathrm{b}}$ & $73.8^{\mathrm{c}}$ \\
\hline
\end{tabular}

Different letters indicate significant differences among treatments within the same column according to least significant difference test $(\mathrm{P}=0.05)$.

TABLE 5. Mean reduction percentages of B. tabaci immature stages on potato plants during season of 2018/2019

\begin{tabular}{|c|c|c|c|c|c|c|c|c|c|c|c|c|}
\hline \multirow{4}{*}{ Insecticides } & \multicolumn{12}{|c|}{$\%$ Reduction after different times of treatment } \\
\hline & \multicolumn{5}{|c|}{$1^{\text {st }}$ spray } & \multicolumn{5}{|c|}{$2^{\text {nd }}$ spray } & \multicolumn{2}{|c|}{$\begin{array}{c}\text { General mean of } 1^{s} \\
\text { and } 2^{\text {nd }} \text { spray }\end{array}$} \\
\hline & \multirow{2}{*}{$\begin{array}{c}1 \text { day } \\
\text { (initial) }\end{array}$} & \multicolumn{3}{|c|}{ Residual } & \multirow{2}{*}{$\begin{array}{l}\text { Mean of } \\
\text { residual }\end{array}$} & \multirow{2}{*}{$\begin{array}{c}1 \text { day } \\
\text { (initial) }\end{array}$} & \multicolumn{3}{|c|}{ Residual } & \multirow{2}{*}{$\begin{array}{l}\text { Mean of } \\
\text { residual }\end{array}$} & \multirow{2}{*}{$\begin{array}{c}1 \text { day } \\
\text { (initial) }\end{array}$} & \multirow{2}{*}{ Residual } \\
\hline & & 3 day & 7 day & 10 day & & & 3 day & 7 day & 10 day & & & \\
\hline Etofenprox & $73.1^{\mathrm{bc}}$ & 74.2 & 89.1 & 55.3 & $72.9^{c}$ & $79.9^{\mathrm{ab}}$ & 66.2 & 75.6 & 83.2 & $75.0^{\mathrm{c}}$ & $76.5^{\mathrm{bc}}$ & $73.9^{\mathrm{c}}$ \\
\hline Pymetrozine & $79.0^{\mathrm{b}}$ & 84.0 & 76.2 & 63.0 & $74.4^{\mathrm{c}}$ & $87.0^{\mathrm{a}}$ & 84.0 & 86.2 & 75.8 & $82.0^{\mathrm{ab}}$ & $83.0^{\text {ab }}$ & $78.2^{\mathrm{bc}}$ \\
\hline Emamectinbenzoate+Thiamethoxam & $60.1^{\mathrm{c}}$ & 91.0 & 83.0 & 83.1 & $85.7^{\mathrm{ab}}$ & $83.7^{\mathrm{ab}}$ & 91.7 & 76.6 & 74.1 & $80.8^{\mathrm{ab}}$ & $71.9^{c}$ & $83.3^{\mathrm{ab}}$ \\
\hline Thiamethoxam & $72.2^{\mathrm{bc}}$ & 85.9 & 90.4 & 68.0 & $81.4^{\mathrm{bc}}$ & $84.2^{\mathrm{ab}}$ & 78.9 & 73.9 & 82.6 & $78.5^{\mathrm{abc}}$ & $78.2^{\mathrm{abc}}$ & $79.9^{\mathrm{ab}}$ \\
\hline Fenitrothion & $91.6^{\mathrm{a}}$ & 97.9 & 88.6 & 83.5 & $90.0^{\mathrm{a}}$ & $77.2^{\mathrm{b}}$ & 71.4 & 72.1 & 85.7 & $76.4^{\mathrm{bc}}$ & $84.4^{\mathrm{a}}$ & $83.2^{\mathrm{a}}$ \\
\hline Imidacloprid & $70.5^{\text {bc }}$ & 88.6 & 89.3 & 81.8 & $86.6^{\mathrm{ab}}$ & $81.5^{\mathrm{ab}}$ & 89.9 & 77.5 & 83.0 & $83.5^{\mathrm{a}}$ & $76.0^{\mathrm{bc}}$ & $85.0^{\mathrm{a}}$ \\
\hline
\end{tabular}

Different letters indicate significant differences among treatments within the same column according to least significant difference test $(\mathrm{P}=0.05)$.

Effect of tested insecticides on some important plant defense parameters

Most of studies focused on the effects ofpesticides on target and non-target pests but little research has focused on the direct effects of insecticides on plants. Here we demonstrate the effect of applications of insecticides on some important plant defense parameters.

\section{Dry matter content of potato leaves}

results of the effect of the six tested insecticides on dry matter percentages of potato leaves were recorded in Table 6 . Giving the impact of the tested insecticides, it was found that all treatments reduced the percentage of dry matter in potato leaves, except for a pesticide pymetrozine which had no significant effect, either by increase or decrease, and these effects were for both the first and the second seasons.

Total soluble protein content of potato leaves

Chemical defense against herbivores is of utmost importance for plants. Primary and secondary metabolites, including non-protein amino acids, have been implicated in plant defense against insect pests (Huang et al., 2011 and van Loon et al., 2006). Among the promising results obtained is that all treatments increased the protein content of potato leaves, whether in the first season or in the second season, and the first place in this increase was a compound imidacloprid which provided the protein content by $3470.11 \mu \mathrm{g}$ protein/gm fresh wt. leaves with $132.45 \%$ of control and $3516.09 \mu \mathrm{g}$ protein/gm fresh wt. leaves with $149.67 \%$ of control in the first and second season, respectively (Table 7).

\section{Total phenols content of potato leaves}

The most commongroup of defensive compounds are phenolic compounds. They are important in the resistance strategy against insects (Sharma et al., 2009 and War et al., 2011).

Table 8 showed that all treatments had a significant increase in the content of potato leaves of phenols, which was estimated as micrograms of tannic acid per gram of fresh potato leaves, and the first place in this increase was the treatment of Emamectin benzoate+Thiamethoxam with an increase of 137.93 and $138.28 \%$ of the control, as a general average for the first and second seasons, respectively. 
TABLE 6. Effect of tested insecticides on \% of dry matter content of potato leaves

\begin{tabular}{|c|c|c|c|c|c|c|}
\hline \multirow{3}{*}{ Insecticides } & \multicolumn{6}{|c|}{$\%$ of dry matter content } \\
\hline & \multicolumn{3}{|c|}{ First season } & \multicolumn{3}{|c|}{ Second season } \\
\hline & $1^{\text {st }}$ Spray & $2^{\text {nd }}$ Spray & Mean & $1^{\text {st Spray }}$ & $2^{\text {nd }}$ Spray & Mean \\
\hline Etofenprox & $31.9 \mathrm{bc}$ & $40.8 b$ & $36.3 b$ & $29.2 \mathrm{~cd}$ & $39.7 \mathrm{~cd}$ & $34.5 \mathrm{c}$ \\
\hline Pymetrozine & $41.1 \mathrm{a}$ & $44.2 \mathrm{ab}$ & $42.7 \mathrm{a}$ & $41.4 \mathrm{a}$ & $46.1 \mathrm{ab}$ & $43.8 \mathrm{a}$ \\
\hline $\begin{array}{l}\text { Emamectin } \\
\text { benzoate+Thiamethoxam }\end{array}$ & $36.4 \mathrm{ab}$ & $39.03 b$ & $37.7 b$ & $36.9 b$ & $38.9 \mathrm{~d}$ & $37.9 \mathrm{~b}$ \\
\hline Thiamethoxam & $31.4 \mathrm{bc}$ & $41.3 b$ & $36.4 \mathrm{~b}$ & $30.8 \mathrm{c}$ & $41.2 \mathrm{~cd}$ & $35.98 \mathrm{bc}$ \\
\hline Fenitrothion & $35.41 \mathrm{ab}$ & $39.8 b$ & $37.6 \mathrm{~b}$ & $35.9 b$ & $40.03 \mathrm{~cd}$ & $37.9 \mathrm{~b}$ \\
\hline Imidacloprid & $27.2 \mathrm{c}$ & $42.96 \mathrm{~b}$ & $35.1 \mathrm{~b}$ & $26.6 \mathrm{~d}$ & $43.02 \mathrm{bc}$ & $34.8 \mathrm{c}$ \\
\hline Control & $37.52 \mathrm{ab}$ & $49.4 \mathrm{a}$ & $43.5 \mathrm{a}$ & $37.8 \mathrm{ab}$ & $47.4 \mathrm{a}$ & $42.6 \mathrm{a}$ \\
\hline
\end{tabular}

Different letters indicate significant differences among treatments within the same column according to least significant difference test $(\mathrm{P}=0.05)$.

TABLE 7. Effect of insecticides on total soluble protein as $\mu \mathrm{g}$ protein/gm fresh wt. potato leaves

\begin{tabular}{|c|c|c|c|c|c|c|}
\hline \multirow{3}{*}{ Insecticides } & \multicolumn{6}{|c|}{$\mu \mathrm{g}$ protein/gm fresh wt. leaves } \\
\hline & \multicolumn{3}{|c|}{ First season } & \multicolumn{3}{|c|}{ Second season } \\
\hline & $1^{\text {st }}$ Spray & $2^{\text {nd }}$ Spray & Mean & $1^{\text {st }}$ Spray & $2^{\text {nd }}$ Spray & Mean \\
\hline Etofenprox & $2374.71^{\mathrm{c}}$ & $2555.75^{\mathrm{b}}$ & $2465.23^{\mathrm{c}}$ & $2066.67^{e}$ & $2944.25^{\text {bc }}$ & $2505.46^{\mathrm{c}}$ \\
\hline Pymetrozine & $2612.07^{\mathrm{bc}}$ & $3118.39^{\mathrm{ab}}$ & $2865.23^{\mathrm{b}}$ & $2453.45^{\mathrm{de}}$ & $3109.20^{\mathrm{b}}$ & $2781.32^{\mathrm{b}}$ \\
\hline $\begin{array}{l}\text { Emamectin } \\
\text { benzoate+Thiamethoxam }\end{array}$ & $2862.64^{\mathrm{b}}$ & $2725.86^{\mathrm{b}}$ & $2794.25^{\mathrm{b}}$ & $3016.7^{\mathrm{abc}}$ & $2691.38^{\mathrm{c}}$ & $2854.02^{\mathrm{b}}$ \\
\hline Thiamethoxam & $2609.77^{\mathrm{bc}}$ & $2685.63^{b}$ & $2647.70^{\mathrm{bc}}$ & $2883.33^{\mathrm{bc}}$ & $2972.99^{\mathrm{bc}}$ & $2928.16^{\mathrm{b}}$ \\
\hline Fenitrothion & $2895.98^{\mathrm{b}}$ & $3717.24^{\mathrm{a}}$ & $3306.61^{\mathrm{a}}$ & $3183.33^{\mathrm{ab}}$ & $3882.76^{\mathrm{a}}$ & $3533.05^{\mathrm{a}}$ \\
\hline Imidacloprid & $3338.51^{\mathrm{a}}$ & $3601.72^{\mathrm{a}}$ & $3470.11^{\mathrm{a}}$ & $3313.22^{\mathrm{a}}$ & $3718.97^{\mathrm{a}}$ & $3516.09^{\mathrm{a}}$ \\
\hline Control & $2763.79^{b}$ & $2499.43^{\mathrm{b}}$ & $2631.61^{\mathrm{bc}}$ & $2715.52^{\mathrm{cd}}$ & $2097.13^{d}$ & $2406.32^{\mathrm{c}}$ \\
\hline
\end{tabular}

Different letters indicate significant differences among treatments within the same column according to least significant difference test $(\mathrm{P}=0.05)$.

TABLE 8. Effect of insecticides on total phenols as $\mu \mathrm{g}$ tannic acid/gm fresh wt. potatoleaves

\begin{tabular}{|c|c|c|c|c|c|c|}
\hline \multirow{3}{*}{ Insecticides } & \multicolumn{6}{|c|}{$\mu g$ Tannic acid } \\
\hline & \multicolumn{3}{|c|}{ First season } & \multicolumn{3}{|c|}{ Second season } \\
\hline & $1^{\text {st'Spray }}$ & $2^{\text {nd }}$ Spray & Mean & $1^{\text {st }}$ Spray & $2^{\text {nd }}$ Spray & Mean \\
\hline Etofenprox & $811.9 \mathrm{de}$ & $1088.6 \mathrm{~b}$ & $950.3 \mathrm{a}$ & $744.4 \mathrm{f}$ & $1099.08 \mathrm{a}$ & $921.8 \mathrm{~d}$ \\
\hline Pymetrozine & $891.1 \mathrm{~cd}$ & $1005.9 \mathrm{c}$ & $948.5 b$ & $875.1 \mathrm{~d}$ & $996.7 \mathrm{~b}$ & $935.9 d$ \\
\hline $\begin{array}{l}\text { Emamectin } \\
\text { benzoate+Thiamethoxam }\end{array}$ & $1145.3 \mathrm{a}$ & $1180.2 \mathrm{a}$ & $1162.7 \mathrm{a}$ & $1135.99 a$ & $1089.9 \mathrm{a}$ & $1112.9 \mathrm{a}$ \\
\hline Thiamethoxam & $783.4 \mathrm{e}$ & $946.2 \mathrm{~cd}$ & $864.8 \mathrm{c}$ & $721.3 \mathrm{f}$ & $916.8 \mathrm{c}$ & $819.03 \mathrm{e}$ \\
\hline Fenitrothion & $1011.8 \mathrm{~b}$ & $998.03 \mathrm{c}$ & $1004.9 \mathrm{~b}$ & $1006.6 \mathrm{~b}$ & $1009.9 b$ & $1008.2 b$ \\
\hline Imidacloprid & $963.6 \mathrm{bc}$ & $997.2 \mathrm{c}$ & $980.4 \mathrm{~b}$ & $933.98 \mathrm{c}$ & $1005.3 b$ & $969.6 \mathrm{c}$ \\
\hline Control & $814.1 \mathrm{de}$ & $873.1 \mathrm{~d}$ & $843.6 \mathrm{c}$ & $807.1 \mathrm{e}$ & $802.50 \mathrm{~d}$ & $804.8 \mathrm{e}$ \\
\hline
\end{tabular}

Different letters indicate significant differences among treatments within the same column according to least significant difference test $(\mathrm{P}=0.05)$. 
Effect of tested insecticides on quality parameters of potato tubers

Pesticide residues may interfere with biochemical and physiological processes in plants retarding the growth and yield of the plant. They may also lower its food quality and prevent its use as food by affecting its quality characteristics (Chauhan et al., 2013). Hence the possible effect of tested insecticides on some quality control parameters were determined.

Effect on potato tuber yield

Based on the yield performance and efficacy against white fly, tuber yield recorded at harvest was subjected tostatistical analysis after converting it into ton per feddan. It revealed that the largest yield in the first season was awarded to the treatment ofThiamethoxam andFenitrothion with yield 5.69 and 4.69 ton per feddan, respectively. The percentage of control reached to $184.85 \%$ for the treatment of insecticide Thiamethoxam. Concerning to the second season, Thiamethoxamretreated to the second place while Imidacloprid occupied the first place, (Figure 1).Our results are consistent with Nag et al., 2018 who found that highest harvested yield of tuber found $129.17 \mathrm{q} / \mathrm{ha}$ on treatmentcombination Imidacloprid and Thiamethoxam. Also, Ghosal and Chatterjee, 2013 observed Imidacloprid gave highest marketable yield. Similar resultswere reported by Afzal et al., 2014 and Ananda et al., 2010.

Total soluble solids (\%TSS) content of potato tubers

The total soluble solids content of the potato tubers as response of six treatments of insecticides, in comparison with untreated control were shown in Figure (2). The results showed that there were no significant differences between the treatments remember the impact on the total soluble solids content especially in the second season. The largest T.S.S was awarded to the treatment of Etofenprox and Emamectin benzoate+Thiamethoxam with a same value of $5.93 \%$ which represents a percentage of control $123.61 \%$.

\section{Ascorbic acid content of potato tubers}

Ascorbic acid (vitamin C) is the main vitamin in potato tubers are also one of the richest sources of antioxidants in the human diet. Figure (3) showed the effect of foliar application of insecticides treatments on ascorbic acid content and untreated control.It was obviously seen that, all the treatments of the first season significantly increased ascorbic acid content of potato tubers and the highest amount of ascorbic acid found was the share of Imidacloprid followed by Fenitrothion with values of 671.16 and 629.24 $\mu \mathrm{g} / \mathrm{gm}$ fresh wt. of potato tubers, respectively. It is evident in the same table that the treatments in the second season took the same trend, as all the treatments provided the content of vitamin $\mathrm{C}$ with a significant increase, and also both Imidacloprid and Fenitrothion retained the first and second places.It is questionable that some research has shown different results from our resultswhere Chauhan et al., 2013 found that the treatment of insecticides decreased the ascorbic acid content of potato tubers.

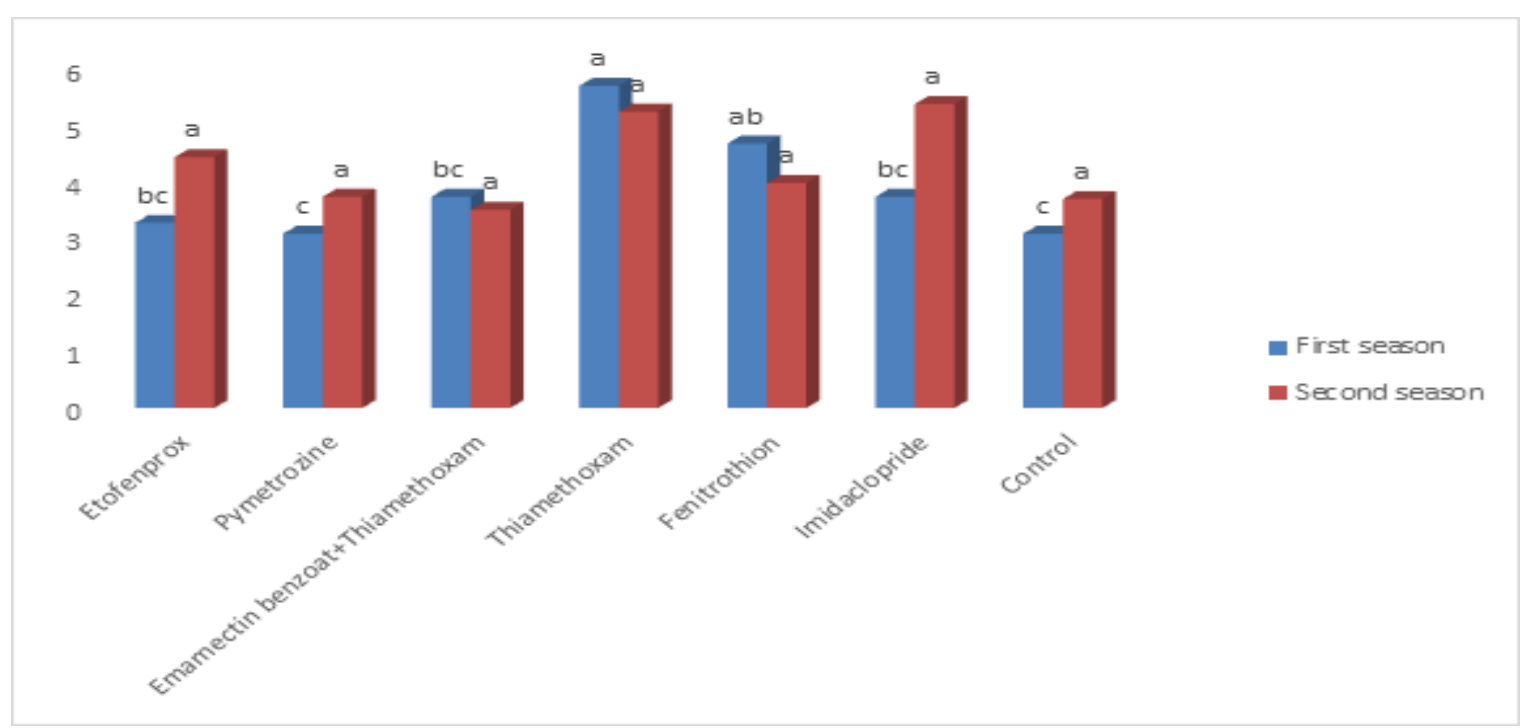

Fig. 1. Effect of tested insecticides on Potato Tuber Yield 


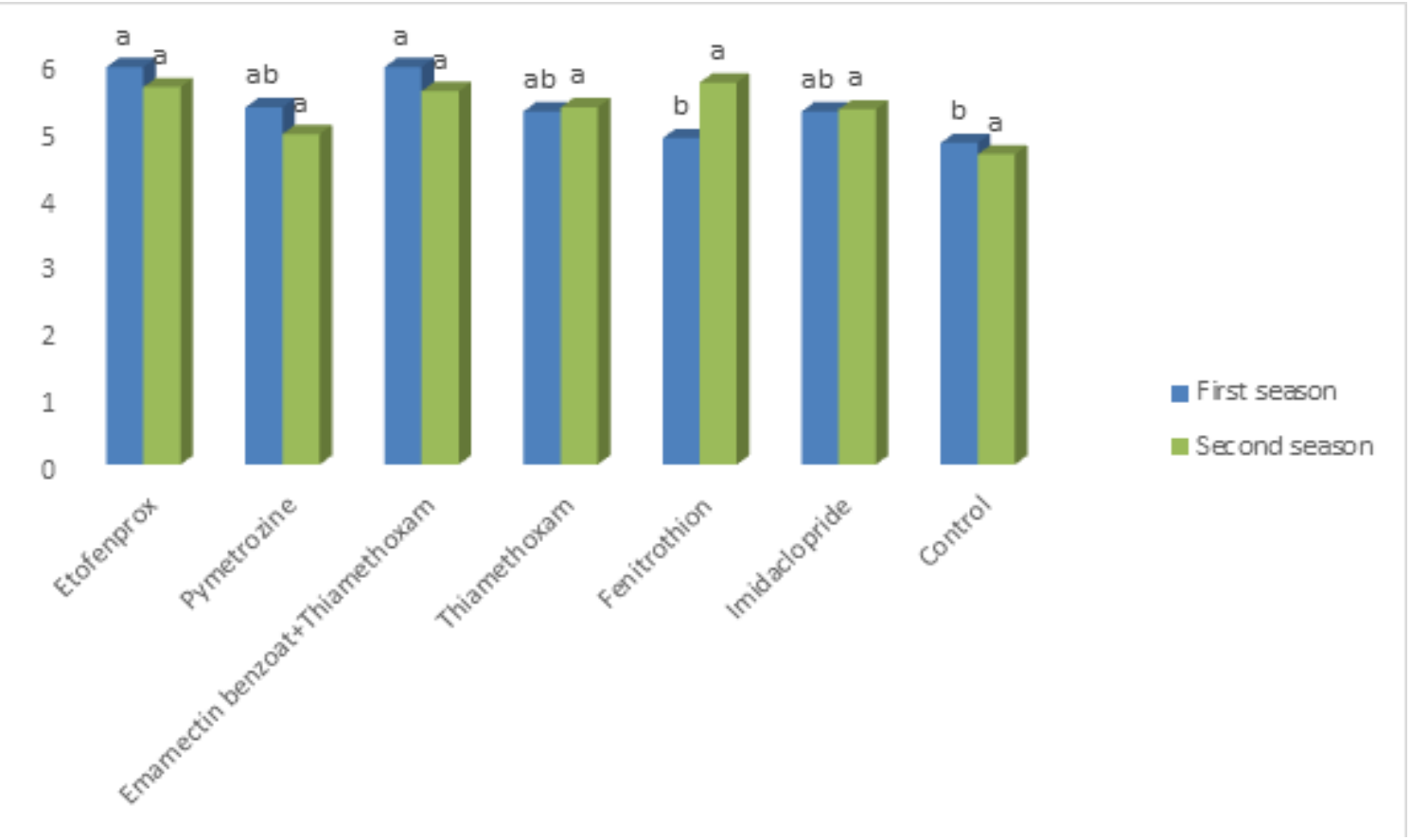

Fig. 2. Effect of tested insecticides on total soluble solids of potato tuber.

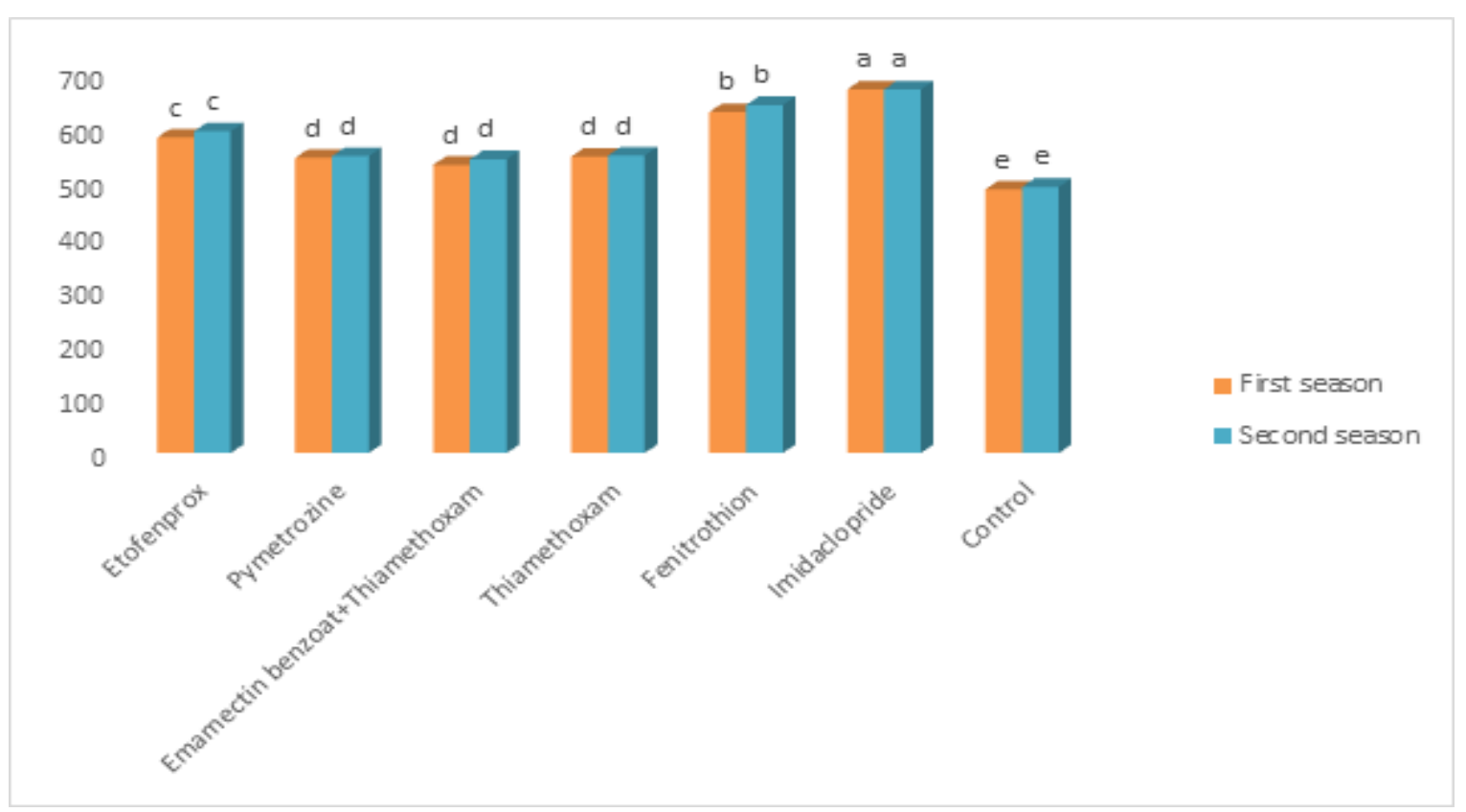

Fig. 3. Effect of tested insecticides on ascorbic acid content of potato tubers

Total acids as citric acid content of potato tubers

Figure 4 shows that all treatments, whether in the first season or in the second season, significantly increased the citric acid content of potato tubers. The highest increase of citric acid percentages in potato tubers were observed in Etofenprox in the first season, Imidacloprid in the second one, since citric acid percentage were 0.187 and $0.182 \%$, respectively.

J. Sus. Agric. Sci. 46, No. 4 (2020)
Total phenol content of potato tubers

The effect of the six treatments of insecticides on the total phenols of potato tubers as $\mu \mathrm{g}$ tannic/gm fresh weightwere shown in Fig. 5. The data revealed that all the treatments either the first season or the second season, significantly increased the total phenol content of the potato. From interestingly, highest total soluble phenols were recorded in Fenitrothionin the two seasons with values of 654.91 and $673.32 \mu \mathrm{g}$ tannic acid/g fresh wt., respectively. 

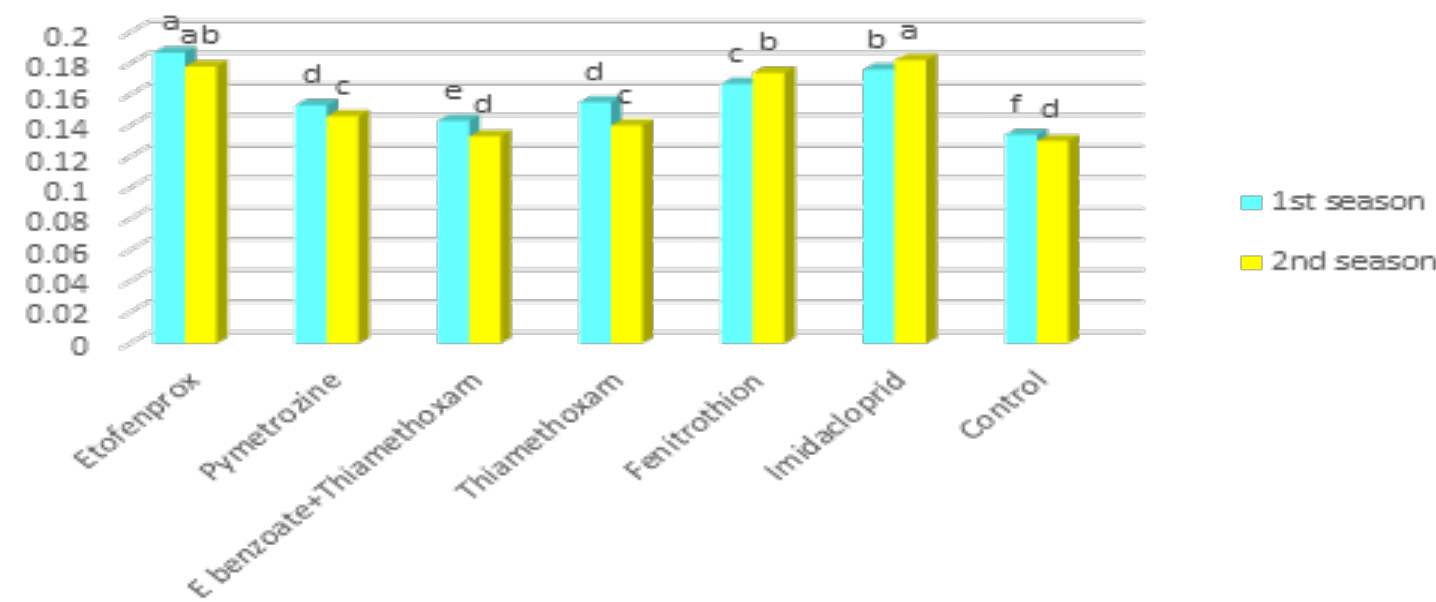

$\square$ 2nd season

Fig. 4. Effect oftested insecticides on citric acid content of potato tubers.

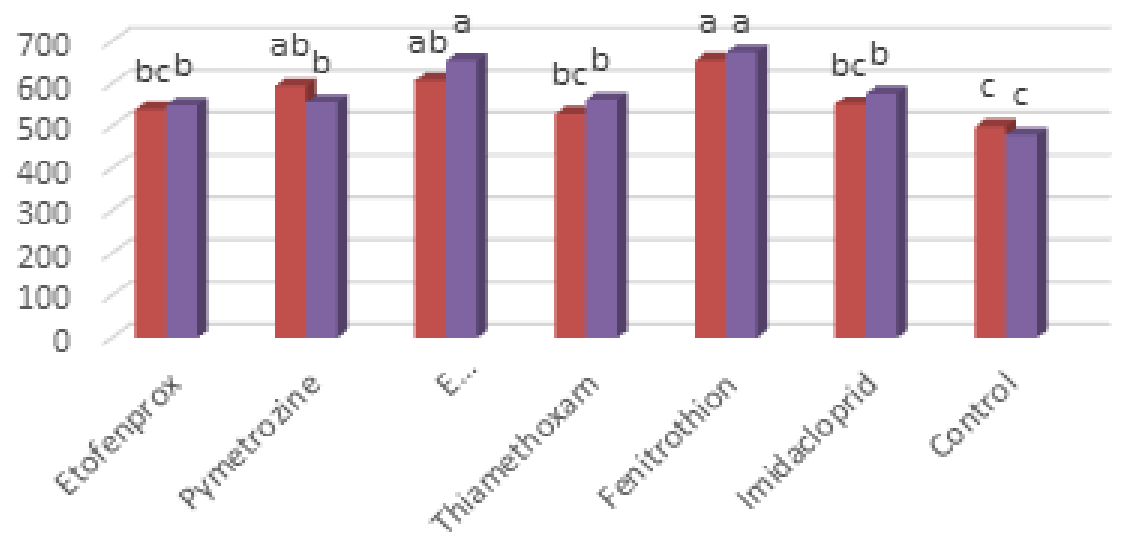

Fig. 5. Effect of tested insecticides on total phenols of Potato Tubers.

Our results differed from some studies since Chauhan et al., (2013) and Hesam et al. (2012) reported a decrease in phenolic content in potatoes by application of pesticide from Iran.

\section{Conclusion}

As general results of the two seasons, it may be concluded that all the insecticidal treatments had highly effect against white fly (Bemisia tabaci) either on adult stage or immature one. And the immature stage was more sensitive to the pesticides used, (Fig. 6).It is really interesting that these insecticides were not satisfied with their direct effect on the white fly, but rather their good effect that they had indirect effects on the plant, as they improved some components of the potato plant that have a role in inducing resistance to insect infestation, (Fig. 7). The effect of insecticides on the insect is a good thing, but it is not surprising, but what is really surprising is to find that it did not negatively affect the plant, but on the contrary, it positively affected the yield, whether in quantity or quality, (Figure 8). The research is a pure work belonging only to its authors and there is no funding for it from any party, as well as there is no conflict between its authors in any way or another.

\section{Author Contributions}

This study was designed and implemented by all authors. They contributed in writing the paper, interpreting information presented and they all have read and agreed to the version of the manuscript.

\section{Conflicts of Interest}

The authors declare that there is no conflict of interest. 


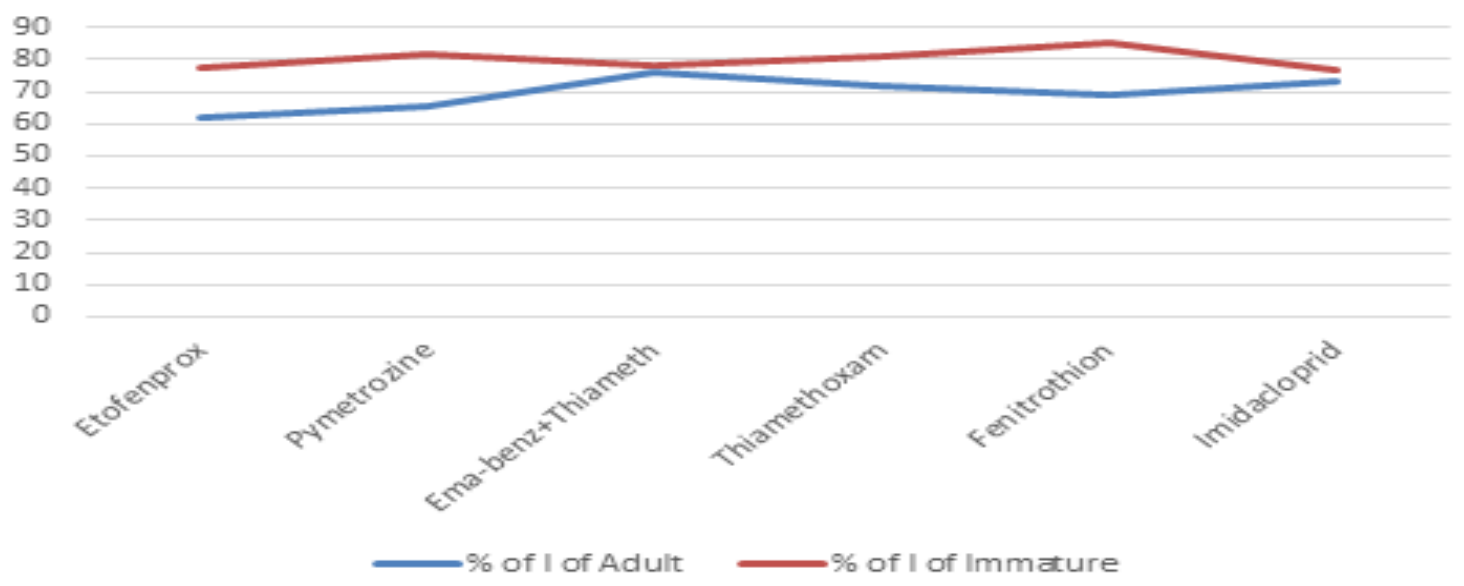

Fig. 6. General mean of Reduction Percentage (\%I) of white fly

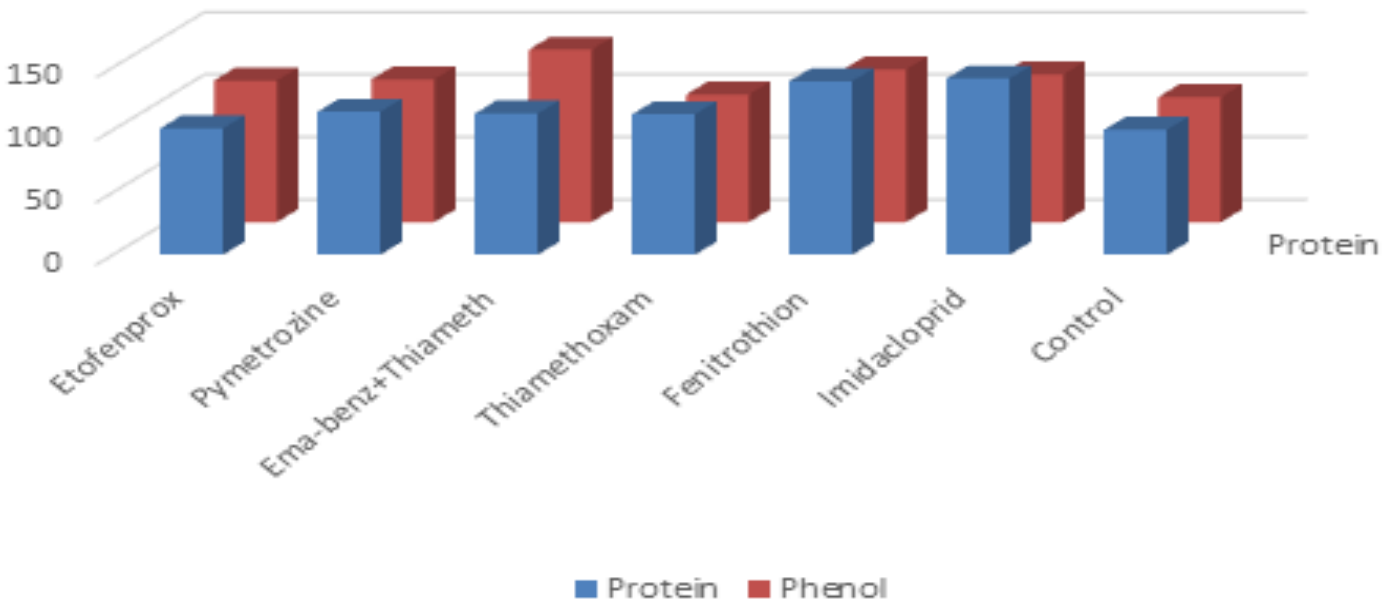

Fig. 7. General effect of Insecticides on Defense Parameters

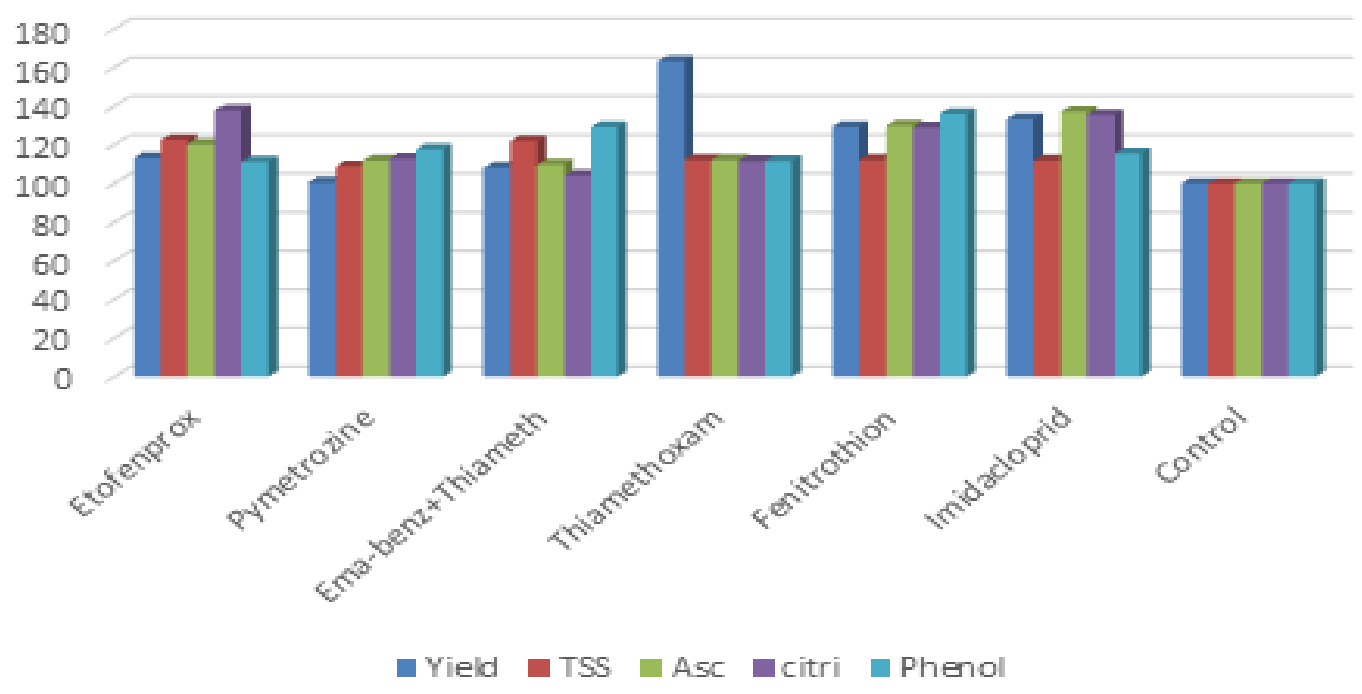

Fig. 8. Effect on Tuber Yield and Quality Parameters

J. Sus. Agric. Sci. 46, No. 4 (2020) 


\section{References}

Abd El-Latif, K. M., Osman, E. A.M., Abdullah, R., and Abd el Kader, N. (2011) Response of potato plants to potassium fertilizer rates and soil moisture deficit. Adv. Appl. Sci. Res., 2 (2), 388-397.

Afzal, M., Rana, S. M., Barbar, M. H. (2014) Comparative Efficacy ofnew Insecticides against Whitefly, Bemisiatabaci (Genn.) and Jassid, Amrascadevastans (Dist.) on Cotton,Bt-121. Biologia (Pakistan), 60 (1), 117-121.

Ahmed, S. M. (2010) Impact of foliar applied fungicides on late blight disease, yield and yield components of threevarieties of potatoes. Journal of Applied Sciences Research, 6 (8), 994-1001.

Ananda, N., Kotikal, Y. K.and Balikai, R. A. (2010) Managementpractices for major sucking pests of pomegranate.Karnataka J. Agril. Sci. 22 (4), 45-49.

Arnemann, J. A.,Bevilaqua, J. G.,Bernardi,L., da Rosa, D. O., da Encarnação,F. A. andPozebon, H. (2019) Integrated Management of Tomato Whitefly Under Greenhouse Conditions. Journal of Agricultural Science; Vol. 11, No. 5, 2019.

Barbehenn, R.V.,Jaros, A., Lee, G.,Mozola, C., Weir, Q. and Salminen, J. P. (2009) Tree resistance to Lymantria dispar caterpillars: importance and limitations of foliar tannin composition. Oecologia 159, 777-788.

Bhatnagar, A. (2007) Incidence and succession of thrips,leafhopper and whitefly in combination of planting datesand potato varieties. Annals. Pl. Protec. Sci. 15,101-105.

Bradford, M.M. (1976) A rapid and sensitive method for the quantification of microgram quantities of protein utilizing the principle of protein-dye binding. Anal. Biochem., 72, 248-254.

Burgos, G., Amoros, W.,Muñoa, L., Sosa, P.,Cayhualla, E., Sanchez, C., Díaz, C. and Bonierbale, M. (2013) Total phenolic, total anthocyanin and phenolicacid concentrations and antioxidant activity of purplefleshed potatoes as affected by boiling. Journal of Food Composition and Analysis. 30, 6-12.

Burgos, G., Auqui, S.,Amoros, W., Salas, E. and Bonierbale, M. (2009) Ascorbic acid concentration of native Andean potato varieties as affected by environment cooking and storage. Journal of Food Composition and Analysis, 22, 533-538.

Butler, G. D.,Coudriet, D. L. and Hennebery, T. V. (1988) Toxicity and repellance of soybeans and cotton seed oils to the sweet potato whitefly and the aphids on cotton in greenhouse studies. Southwest, Entomol. 13, 81-96.

Chandel, R. S.,Banyal, D. K., Singh, B. P., Malik, K. and Lakra, B. S. (2010) Integrated Management of Whitefly, Bemisiatabaci (Gennadius) and Potato Apical Leaf Curl Virus in India Potato Research. 53, 129-139

Chauhan, S.S., Agrawa, S.and Srivastava, A. (2013) Effect of imidacloprid insecticide residue on biochemical parameters in potatoes and its estimation by HPLC. Asian J Pharm Clin Res, Vol 6, Supp 13, 114-117.

CoStat Statistical Software. (1990) Microcomputer program analysis version 4. 20, Co Hort Software, Berkeley, CA.

Dixon, R.A. (1985) Plant cell culture, a practical approach IRLPRESS. Oxford. Washington DC. 1-235.

Egoaville, M., Sullivan, J.,Kozempel, M. and Jones, W. (1988) Ascorbic acid determination in processed potatoes. American Potato Journal. 65, 91-97.

El-Naggar, J.B. and Zidan, L.T.M. (2013) Field evaluation of imidacloprid and thiamehoxam against sucking insects and their side effects on soil fauna. J. Plant Protection Research. 53 (4), 375 386.

Eyles, A., Bonello,P., Ganley, R. and Mohammed, C. (2010) Induced resistance to pests andpathogens in trees. 4,5,6 New Phytologist, 185, 893-908doi: 10.1111/j.1469-8137.2009.03127.x.

Ford, K. A.,Casida, J. E., Chandran, D.,Gulevich, A.G., Okrent, R.A. (2010) Neonicotinoid insecticides induce salicylate-associated plant defense responses. Proceedings of the National Academy of Sciences of the United States of America 107, 17527-17532.

Franceschi, V.R., Krokene, P., Christiansen, E. andKrekling, T. (2005) Anatomical and chemical defenses of conifer bark against bark beetles and otherpests. New Phytologist, 167, 353-375.

Ghosal, A. and Chatterjee, M. L. (2013) Bioefficacy of Imidacloprid17.8 SL against whitefly, Bemisiatabaci (Gennadius) inbrinjal. J. Pl. Prot. Sci. 5 (1), 37-41.

Henderson, C. and Tilton, E. (1955) Tests with acaricides against the brown wheat mite. J. Econ. Entomol. 48, 157-161.

J. Sus. Agric. Sci. 46, No. 4 (2020) 
Hesam, F., Balali, G.R. and Tehrani, R. T. (2012) Evaluation of antioxidant activity of three common potato (Solanum tuberosum) cultivars in Iran. Avicenna Journal of Phytomedicine. 2 (2), 79-85.

Hirose, E. Batista, A. S. and Silva, M. S. (2015) Correlação da ocorrência de fumaginaem soja com a população de ninfas de mosca-branca Bemisiatabaci (Hemiptera: Aleyrodidae). Embrapa Soja, Cuiabá, MT: VI Congresso Brasileiro de Soja.

Huang, T., Jander, G. and Martin, V. (2011) Non-protein amino acids in plant defense against insect herbivores: Representative cases and opportunities for further functional analysis. Hytochemistry, 72 (13), 1531-7.

Karthikeyan, G., Doraisamy, S. andRabindran, R. (2009) Induction of systemic resistance in blackgram (Vigna mungo) against urdbean leaf crinkle virus by chemicals. Archives of Phytopathology and Plant Protection, 42, 1-15.

Keeling, C.I. and Bohlmann, J. (2006) Genes, enzymes and chemicals of terpenoiddiversity in the constitutive and induced defence of conifers againstinsects and pathogens. New Phytologist, 170, 657-675.

Kim, G. Y., Lee, I. L. and Ahn, K. (2000) Susceptibility of sweetpotato whitefly,Bemisiatabaci (Homoptera: Aleyrodidae) to commercially registered insecticides in Korea. The Korean Journal of Pesticide Science. 4 (1), 51-58.

Lattanzio, V., Lattanzio, V. M. T. and Cardinali, A. (2006) Role of phenolics in theresistance mechanisms ofplants against fungalpathogens and insects. Phytochemistry: Advances in Research, 2367 ISBN: 81-308-0034-9.

Nag, D., Nag, R., Bhargav, P., Kumar, Y. andBisen, M. S.(2018) Relative Efficacy of Insecticides against PotatoAphid (Myzuspersicae Sulzer), White Fly (Bemisiatabaci Genn.) and Lady Bird Beetle. International Journal of Chemical Studies. 6 (1), 1182-1186.

Nombela, G. andMuñiz,M. (2010) Host plant resistance for the management of Bemisiatabaci: a multicropsurvey with emphasis on tomato. In: Stansly PA, Naranjo SE (Eds) Bemisia: bionomicsand management of a global pest. Springer Science Business Media B.V., pp 357-383.doi:10.1007/97890-481-2460-2.

Palumbo, J. C. (2019) Impact of Sweet Potato Whitefly Infestation on Yield and Quality of Cantaloupe. http://hdl.handle.net/10150/221467.

Preetha, G., Manoharan, T., Stanley, J. andKuttalam, S. (2009) Evaluation of Imidacloprid against okra jassid, Amrascabiguttulabiguttula (Ishida) and whitefly, Bemisiatabaci (Gennadius). Indian J. Ent. 71 (3), 209-214.

J. Sus. Agric. Sci. 46, No. 4 (2020)
Ruuhola, T., Yang, S. Y., Ossipov, V. andHaukioja, E. (2008) Foliar oxidases asmediators of the rapidly induced resistance of mountain birch against Epirritaautumnata. Oecologia, 154, 725-730.

Sabra, F.S. (1993) Studies on the Chemical Weed Control. Studies on the Efficiency of Certain Herbicides and their Side Effect on Potato Plants and Soil. Ph.D. thesis, Faculty of Agric. Alex. University.

Sharma, H. C., Sujana, G., and Rao, D. M. (2009) Morphological and chemicalcomponents of resistance to pod borer, Helicoverpaarmigera in wild relativesof pigeonpea. Arthropod Plant Interact. 3, 151-161. doi: 10.1007/s11829-009-9068-5.

Slinkard, K. and Singleton, V.L. (1997) Total phenol analysis: automation and comparison with manual methods. American Journal of Enology and methods. American Journal of Enology and Viticulture, 28, 49-55. 1997.

Szczepaniec, A., Raupp, M. J., Parker, R. D., Kerns, D. and Eubanks M. D. (2013) Neonicotinoid Insecticides Alter Induced Defenses and Increase Susceptibility toSpider Mites in Distantly Related Crop Plants. PLoS ONE 8 (5), e62620. doi:10.1371/ journal.pone.0062620.

Tsai, C. J., Harding, S. A., Tschaplinski, T. J., Lindroth, R. L.and Yuan YN. (2006) Genome-wide analysis of the structural genes regulating defense phenylpropanoidmetabolism in Populus. New Phytologist 172, 47-62.

Van Loon,L.C., Rep,M. and Pieterse, C.M.J. (2006) Significance of Inducible Defense-related Proteins in Infected Plants. Article in Annual Review of Phytopathology, Vol. 44, 135-162.

War, A.R., Paulraj, M.G., War, M.Y., and Ignacimuthu, S. (2011) Jasmonicacid-mediated-induced resistance in groundnut (Arachis hypogaea L.) against Helicoverpaarmigera (Hubner) (Lepidoptera: Noctuidae). J. Plant GrowthRegul. 30, 512-523.

Waterhouse, A.,(2002)Determination of total phenolics. Current Protocols in Food Analytical Chemistry. University of California, U.S.A. I1.1.1-I.1.1.8.

Witzell, J. and Martin, J. A. (2008) Phenolic metabolites in the resistance ofnorthern forest trees to pathogens - past experiences and future prospects.Canadian Journal of Forest Research 38, 2711-2727.

Zarzecka, K. and Gugata, M. (2011) The effect of herbicides and soil tillage systems on the content poly phenols in potato tuber. Polish J.of Environ. Stud.vol. 20, No. 2, 513-517. 


\section{تقبيم ستة من المبيدات الحشرية لمكافحة ذبابة البيضاء فى البطاطس(Bemisiatabaci)

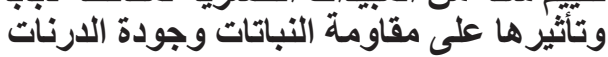

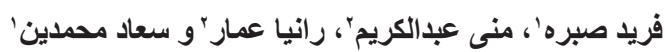

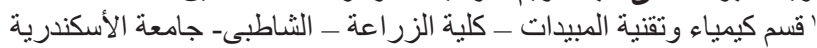

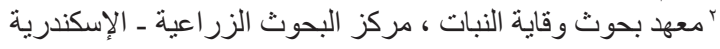

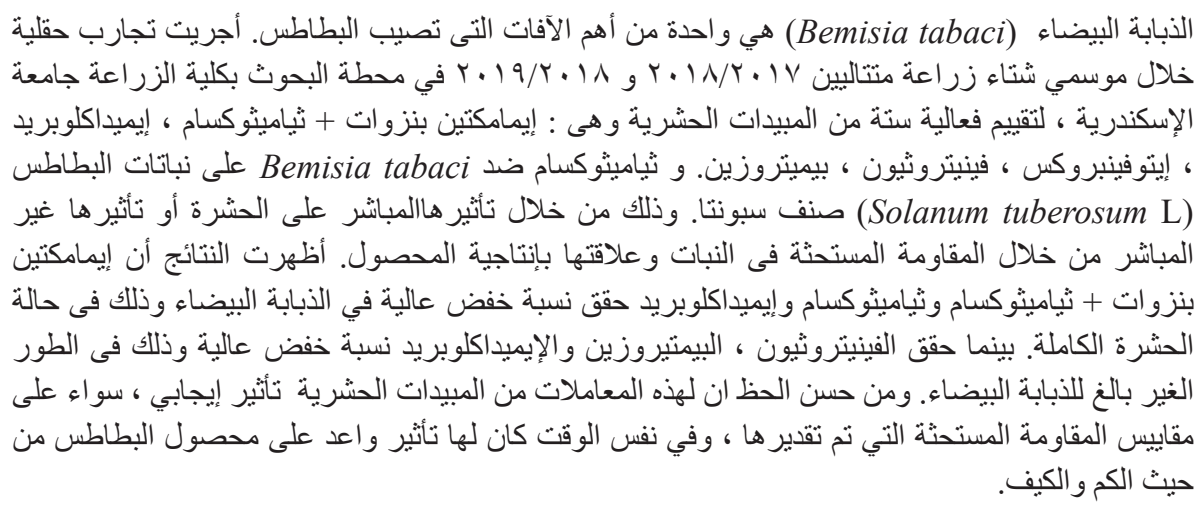

